\title{
Complete Calcified Ureteral Stent: A Less Challenging Scenario When Using Adequate Technology
}

\author{
Rivero Guerra $\mathrm{A}^{* 1}$, Pietricica $\mathrm{BN}^{2}$ and Fernandez Aparicio $\mathrm{T}^{2}$ \\ ${ }^{1}$ Santa Lucia University Hospital, Spain \\ ${ }^{2}$ Hospital Morales Meseguer, Spain
}

Received: 漹: December 18, 2018; Published: 制: January 03, 2019

*Corresponding author: Rivero Guerra A, Santa Lucia University Hospital, Spain

\section{Introduction}

Ureteral stents and nephrostomy tubes are routinely used in many urological procedures. Significant technological innovations and improvements have been developed during the past decades in stent design and material; however, serious complications, including migration, fragmentation, encrustation and stone formation, still may occur [1]. Forgotten stents due to loss of follow-up may undergo severe encrustation and stone formation, a situation for which there is still no consensus on the best therapeutic approach [2].

\section{Case Report}

A 55-year-old man presented to our department in September 2017 with hematuria and lower back pain. He had prior history of bilateral ureteral stenting in a different centre in August 2017 due to bilateral renal obstruction with anuria and acute renal failure (serum creatinine level up to $5.4 \mathrm{mg} / \mathrm{dL}$ ). He had subsequently passed a uric acid stone $<5 \mathrm{~mm}$.

A Non-Contrast CT Scan was Performed $3.5 \times 1 \mathrm{~cm}$ right ureteral stone and bilateral grade 2 hydronephrosis were observed (Figure 1). Removal of both stents and right retrograde Ureteroscopy (URS) were recommended and alkalinisation of urine by application of alkaline citrate was started. URS was scheduled on February 2018. Both stents were unsuccessfully removed due to calcification. Right URS was then carried out. Through a nitinol guidewire, an 8F semirigid ureteroscope under fluoroscopic guidance was introduced into the right ureteral orifice beside the encrusted stent and ascended up to the proximal ureter, where the aforementioned stone was formed around the stent. This stone was fragmented using a $300 \mathrm{~nm}$ Holmium laser and fragments were removed with NGage区 Nitinol Stone Extractor (Cook冈 Medical). The stent was completely extracted afterwards and a new 6F stent was placed under fluoroscopic guidance.

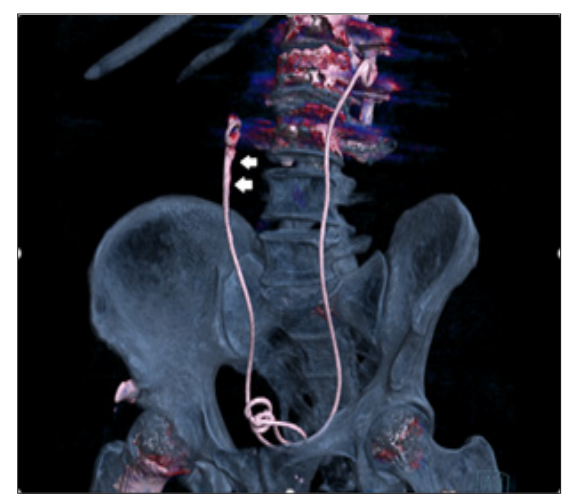

Figure 1: Non-contrast CT scan: Right ureteral stone (arrows).

The same approach was unsuccessfully intended in the left ureter, as the stent was completely calcified from the mid ureter up to the renal pelvis, and the ureteroscope progression from this point could not be achieved. The stent was then cut off in the most proximal reachable point and the distal part was removed. The surgery was then interrupted, and a left nephrostomy tube was placed (Figure 2). Three weeks after, a second surgery was scheduled. First, the right stent was removed. A SensorTM guidewire (Boston Scientific) was inserted through the nephrostomy tube, the tract was dilated up to $18 \mathrm{~F}$ under fluoroscopic guidance and a mini-nephroscope was introduced. The proximal portion of the stent was completely calcified (Figure 3 ). The stone around the stent was fragmented using a 300nm Holmium laser (Figure 4) and fragments were removed with a Zero TipTM Nitinol Stone Retrieval Basket (Boston Scientific). When it was free from encrustations, the remaining portion of the stent was removed using the same nitinol basket. A new ureteral stent was placed and then removed without further incidence after two weeks. A new non-contrast CT scan 
was performed four weeks after the second surgery and stone-free status of the patient was confirmed.

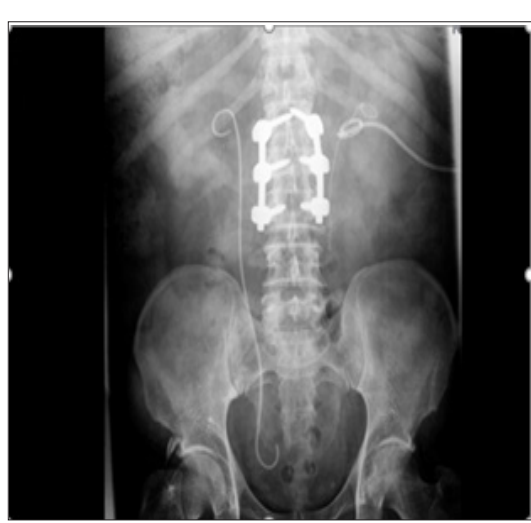

Figure 2: X-Ray. Right ureteral stent left ureteral stent cut off and left nephrostomy tube.

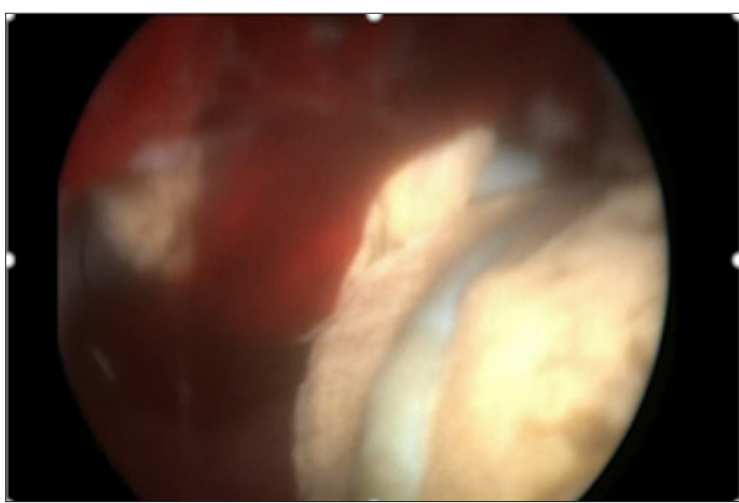

Figure 3: Completely calcified stent, percutaneus approach.

\section{ISSN: 2574-1241}

DOI: 10.26717/BJSTR.2019.12.002298

Rivero Guerra A. Biomed J Sci \& Tech Res

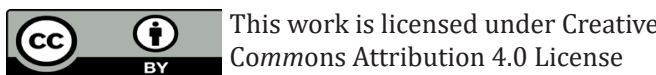

Submission Link: https://biomedres.us/submit-manuscript.php

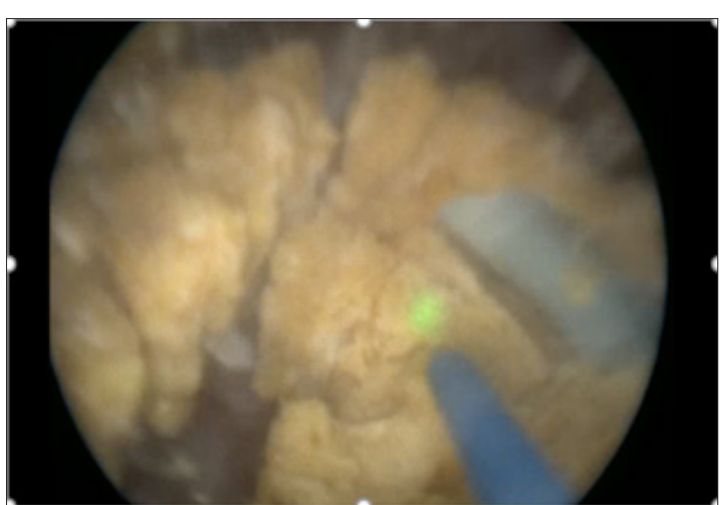

Figure 4: Holmium laser fragmentation of calcification around the stent.

\section{Conclusion}

Encrusted/calcified stents represent an unwanted and problematic scenario for urologists, although a much less challenging one when a wide range of endourology equipment and technology is available. Education to patients in order to avoid forgotten stents is still the best way of preventing this complication.

\section{References}

1. Xu C, Tang H, Gao X, Yang B (2009) Management of forgotten ureteral stents with holmium laser. Lasers Med Sci 24(2): 140-143.

2. Saha PK, Hossain MS, Ghosh KC, Alam MS, Nabi S, et al. (2018) Forgotten, Encrusted Ureteral Stents: Removal - Multimodal Endourologic Approach. Mymensingh Med J 27(1): 149-158.

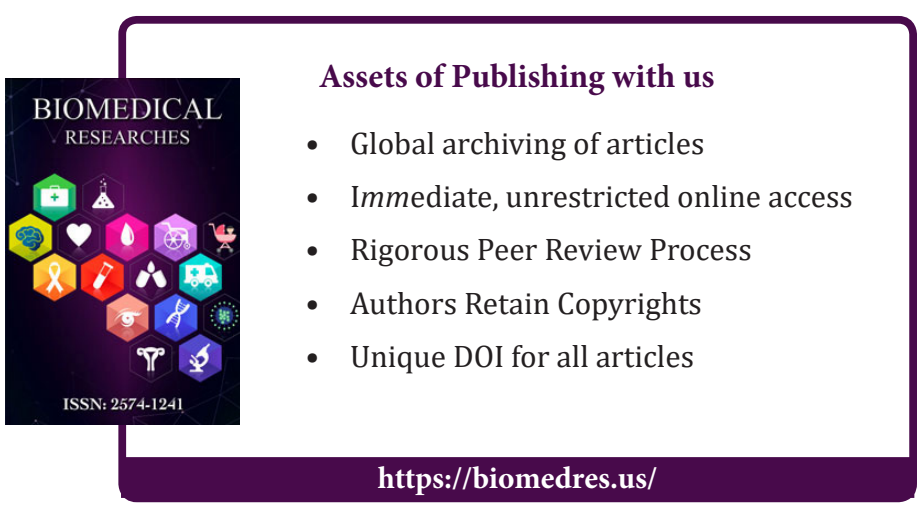

